\title{
Hyperon softening of the EOS of dense matter and the spin evolution of isolated neutron stars
}

\author{
J. L. Zdunik ${ }^{1}$, P. Haensel ${ }^{1,2}$, E. Gourgoulhon ${ }^{2}$, and M. Bejger ${ }^{1}$ \\ 1 N. Copernicus Astronomical Center, Polish Academy of Sciences, Bartycka 18, 00-716 Warszawa, Poland \\ e-mail: bejger@camk.edu.pl \\ 2 LUTH, UMR 8102 du CNRS, Observatoire de Paris, 92195 Meudon Cedex, France \\ e-mail: haensel@camk.edu.pl; Eric.Gourgoulhon@obspm.fr
}

Received 24 September 2003 / Accepted 12 November 2003

\begin{abstract}
The effect of the hyperon softening of the equation of state (EOS) of dense matter on the spin evolution of isolated neutron stars is studied for a broad set of hyperonic EOSs. We use a multidomain 2-D code based on a spectral method, and show how important the precision of solving the equations of stationary motion is for the stability analysis. For some EOSs, the hyperon softening leads to spin-up by angular momentum loss, in the form of the back-bending phenomenon, for a rather broad range of stellar baryon mass. We show that large segments of the evolutionary tracks exhibiting the back-bending behaviour in the moment-of-inertia - rotation-frequency plane are unstable and therefore not astrophysically relevant. We show also that during the spin-up - angular-momentum-loss epoch, an isolated neutron star (e.g. a radio pulsar) can lose a sizable part of its initial angular momentum without significantly changing its rotation period. We propose also simple arguments and criteria allowing one to connect the presence of a back-bending epoch with the mass-radius relations and the stiffness and/or softness of the nucleon and hyperon EOSs of the neutron star core.
\end{abstract}

Key words. dense matter - equation of state - stars: neutron - stars: rotation

\section{Introduction}

Some theories of dense matter predict a softening of the equation of state (EOS) at densities exceeding normal nuclear density $\rho_{0}=2.7 \times 10^{14} \mathrm{~g} \mathrm{~cm}^{-3}$, implied by a phase transition to a new "exotic" phase. Several "exotic" high-density phases were proposed in the past, e.g., deconfined quark plasma, and pioncondensed or kaon-condensed hadronic matter. The softening of the EOS could be due to a transition into a pure "exotic" phase, or to a mixture of an "exotic" phase with normal phase of dense matter. It has been suggested that the softening of the EOS due to a phase transition could lead to characteristic backbending phenomenon in the timing of spinning-down pulsars (Glendenning et al. 1997) or produce characteristic period clustering in spinning-up neutron stars in low-mass $\mathrm{X}$-ray binaries (Glendenning 2001; Glendenning \& Weber 2002). An interesting conclusion of these papers was that both back-bending in spinning down pulsar and spin clustering in accreting millisecond pulsars stars is evidence of a phase transition taking place at the center of a spinning-down pulsar or a spinning-up accreting neutron star.

As we show in the present paper, using several EOS of dense matter and very precise code for the calculation of the rotating stellar configurations, observation of the back-bending

Send offprint requests to: J. L. Zdunik, e-mail: jlz@camk.edu.pl in the timing behavior of isolated pulsars, or of the period clustering, is not unambiguous evidence for an "exotic" phase in dense matter. These phenomena can also be implied by the presence of hyperons in dense matter, a feature which is in no way "exotic" and which was predicted more than forty years ago (Cameron 1959; Salpeter 1960; Ambartsumyan \& Saakyan 1960). A possibility of "spin-up by the angular momentum loss" for a normal sequence (baryon mass smaller than the maximum baryon mass of static configurations) of spinningdown neutron stars with hyperonic cores was previously noted by Balberg et al. (1999). However, as we will demonstrate in the present paper, a complete study of back-bending requires a very precise "exact" code for calculating stationary configurations of rotating stars and a simultaneous careful checking of secular stability of these configurations. Such conditions were typically not satisfied in previous calculations.

In previous works, the back-bending phenomenon has been considered as a feature of the $I(\Omega)$ dependence, where $I$ is the moment of inertia of the star and $\Omega$ is the angular frequency of rotation (Glendenning et al. 1997; Cheng et al. 2002; Spyrou \& Stergioulas 2002). In the present paper we clarify some statements about the back-bending for rotating neutron stars, and we formulate some rules which are useful for searching for the back-bending in rotational stellar sequences. As we demonstrate using high-precision evolutionary sequences, the whole 
analysis should be performed using different pair of variables: total stellar angular momentum $J$ versus $\Omega$ instead of $I(\Omega)$. High precision is particularly important because it is needed to reliably check the secular stability of rotating configurations; only the stable ones are interesting and observationally relevant.

The paper is organized in the following way. Softening of the EOS due to the presence of hyperons is discussed in Sect. 2. The method allowing for a high precision of the 2-D calculations of the equilibrium configurations of rotating neutron stars is briefly described in Sect. 3. Different formulations of the stability criteria for rotating configurations are briefly summarized in Sect. 4. In Sect. 5 we propose a method of checking for the occurrence of the back-bending phenomenon by inspecting the baryon-mass - equatorial-radius relations at fixed values of rotation frequencies. We apply this method to several EOSs with a hyperon softening. The interplay between the back-bending and stability is discussed in Sect. 6, where we study neutron-star evolution tracks in the angular-momentum rotation-frequency plane. In Sect. 8 we study the dependence of the back-bending phenomenon on the EOS of the hyperonic matter. Section 9 contains discussion of our results, including their possible observational aspects, and concluding remarks.

\section{Equation of state with hyperons}

Possible presence of hyperons in dense neutron-star matter is mainly the consequence of the Pauli principle for neutrons and electrons, which at a sufficiently high baryon density can make a replacement of high-energy neutrons and electrons by more massive but slower hyperons energetically favorable. Hyperon species $\mathrm{H}$ is then present above a certain threshold $\rho_{\mathrm{H}}$, which is determined by a condition involving the change of energy of dense matter, due to addition of a single hyperon, at a fixed pressure, $\mu_{\mathrm{H}}^{0}$. The threshold density for $\mathrm{H}$ is the lowest density at which the equality

$\mu_{\mathrm{H}}^{0}=\mu_{\mathrm{n}}-q_{\mathrm{H}} \mu_{\mathrm{e}}$,

is satisfied. Here, $\mu_{\mathrm{n}}$ and $\mu_{\mathrm{e}}$ are the chemical potentials of neutrons and electrons (which include rest energies), and $q_{\mathrm{H}}$ is the hyperon charge in units of $e$. The threshold condition given by Eq. (1) shows that because of the high value of $\mu_{\mathrm{e}}$, hyperons with negative charge are strongly preferred over the positively charged ones. It also explains, why $\Sigma^{-}$, and not the lowest mass hyperon, $\Lambda^{0}$, is usually the first hyperon to appear in dense matter. It should be stressed, however, that the hyperon-nucleon interactions which contribute significantly to $\mu_{\mathrm{H}}^{0}$, are poorly known, and this implies uncertainty not only in the values of $\rho_{\mathrm{H}}$ but also in the order of the hyperon appearance. Let us notice that for some models of dense matter hyperons do not appear in neutron stars at all, because the lowest $\rho_{\mathrm{H}}$ is larger than the maximum density in neutron stars (see, e.g., Pandharipande \& Garde 1972).

The hyperon softening is particularly well visualized by the behavior of the adiabatic index

$\Gamma=\frac{n_{\mathrm{b}}}{P} \frac{\mathrm{d} P}{\mathrm{~d} n_{\mathrm{b}}}$.

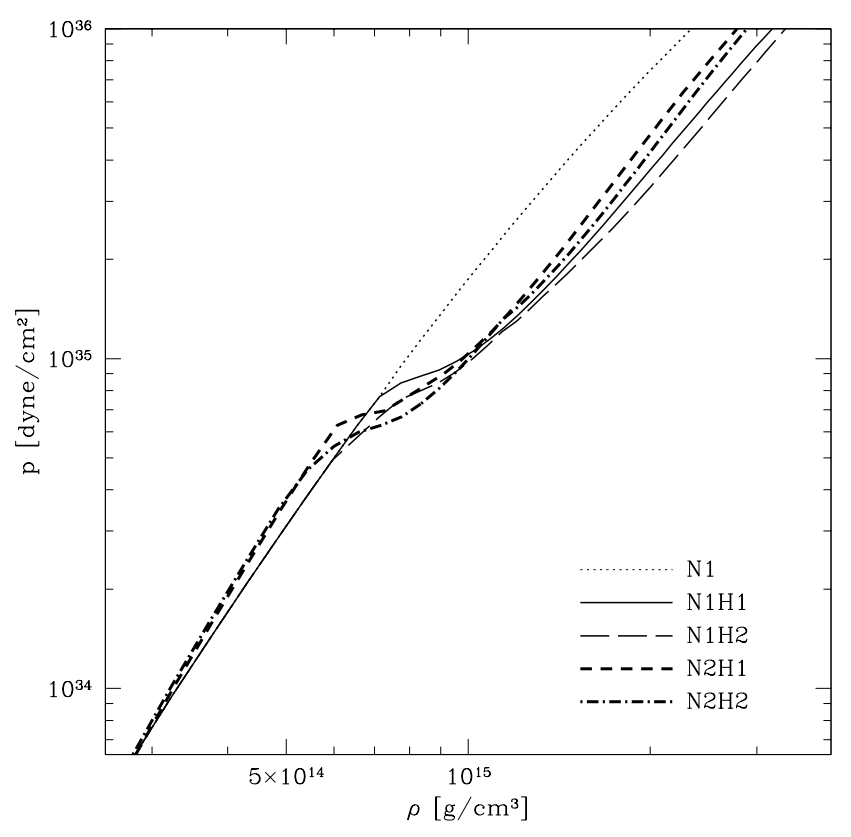

Fig. 1. Equations of state of hyperonic matter calculated by Balberg \& Gal (1997); and used in this paper. Our notation is a close analogue of that introduced by Balberg et al. (1999). Our labels N1, N1H1, and $\mathrm{N} 1 \mathrm{H} 2$ are their EoS1 N, EoS1 N$\Lambda \Xi$, and EoS1 N $\Lambda \Sigma \Xi$, respectively. Our $\mathrm{N} 2 \mathrm{H} 1$ and $\mathrm{N} 2 \mathrm{H} 2$ are their EoS2 $\mathrm{N} \Lambda \Xi$ and $\operatorname{EoS} 2 \mathrm{~N} \Lambda \Sigma \Xi$, respectively. For further explanations see the text.

At each threshold density $\rho=\rho_{\mathrm{H}}$ the function $\Gamma(\rho)$ suffers a drop. For some models, typical values of $\Gamma \simeq 2-3$ characteristic of the nucleonic matter at $\rho \sim(2-4) \rho_{0}$ can drop even down to $\Gamma \sim 1$ (see, e.g., Balberg et al. 1999). For other models the drop is not so dramatic but still sizable (see, e.g., Haensel et al. 2002). The softening of the EOS by hyperonization of matter can be also visualized by comparing the values of the maximum allowable mass for non-rotating neutron stars, $M_{\max }$, for the EOS without hyperons, referred hereafter as the N EOS, and those involving nucleons and hyperons (NH). Typically, allowing for the presence of hyperons lowers the value of $M_{\max }$ by $(0.3-0.6) M_{\odot}$ (see, e.g., Haensel 2003). The presence of hyperons leads to a very characteristic flattening of the massradius and mass-central density plot for neutron star, with a knee taking place just after the threshold for the first hyperon (Glendenning 1985; Balberg et al. 1999). This feature will be important in the context of the back-bending phenomenon in rotating neutron stars.

In present paper we use the EOSs calculated by Balberg $\&$ Gal (1997). These EOSs are presented in Fig. 1. They are based on phenomenological effective baryonic matter energy functionals, resulting from effective baryon-baryon interactions whose parameters are adjusted to reproduce the empirical properties of nuclear matter as well as the basic experimental features of the hyperonic interactions. Two models of nucleon matter, based on an effective nucleon-nucleon (NN) interaction, constructed by Balberg \& Gal (1997) lead to the EOSs N1 and $\mathrm{N} 2$ of dense matter without hyperons (they correspond to their models EoS1 N and EoS2 N, in the notation of Balberg et al. 1999). The model N1 corresponds to the incompressibility of nuclear matter at saturation $K_{0}=240 \mathrm{MeV}$ (i.e., is close to 
the experimental value of this parameter). The model N2 gives $K_{0}=320 \mathrm{MeV}$, and leads to a significantly stiffer EOS than the N1 one. Balberg \& Gal constructed also two models representing effective nucleon-hyperon $(\mathrm{NH})$ and hyperon-hyperon $(\mathrm{HH})$ interactions in baryonic matter. We will denote them by $\mathrm{H} 1$ and $\mathrm{H} 2$; they correspond to the $\Lambda \Xi$ and $\Lambda \Sigma \Xi$ effective hyperon interaction models of Balberg \& Gal (1997) in the notation of Balberg et al. (1999). Model H2 leads to a stronger softening of the EOS due to the presence of hyperons than the $\mathrm{H} 1$ one. The EOS of baryonic matter are obtained by combining $\mathrm{NN}, \mathrm{NH}$, and $\mathrm{HH}$ effective interactions. In this way one obtains four EOSs of baryonic matter with hyperons. We will denote them by $\mathrm{N} 1 \mathrm{H} 1, \mathrm{~N} 1 \mathrm{H} 2, \mathrm{~N} 2 \mathrm{H} 1$, and $\mathrm{N} 2 \mathrm{H} 2$; they correspond to $\operatorname{EoS} 1 \mathrm{~N} \Lambda \Xi, \operatorname{EoS} 1 \mathrm{~N} \Lambda \Sigma \Xi, \operatorname{EoS} 2 \mathrm{~N} \Lambda \Xi$ and $\operatorname{EoS} 2 \mathrm{~N} \Lambda \Sigma \Xi$ in the notation of Balberg et al. (1999), respectively. For the sake of completeness, we considered also five EOSs of Glendenning (1985); see Sects. 8 and 9.

\section{Numerical calculations}

The neutron star models have been computed in full general relativity by solving the Einstein equations for stationary axi-symmetric spacetime (see e.g. Bonazzola et al. 1993 or Gourgoulhon et al. 1999 for the complete set of partial differential equations to be integrated). The numerical computations have been performed via the Lorene/Codes/Rot_star/rotstar code from LORENE (http://www. lorene.obspm.fr/). This C++ code implements a multi-domain spectral method introduced in Bonazzola et al. (1998). A description of the code can be found Gourgoulhon et al. (1999). For the purpose of the present work, we have employed two domains to describe the neutron star interior, making use of the adaptive coordinates to set the boundary between the innermost domain and the outer one at the transition surface to hyperon matter. In this way, the density field is smooth in each domain and the spectral method results in a high accuracy. This accuracy has been checked by evaluating the GRV2 and GRV3 virial error indicators (see Nozawa et al. 1998), which showed a relative error lower than $\sim 10^{-5}$.

The physical parameters resulting from the equation of state (pressure, energy density, number density) are obtained by the Hermite interpolation (Swesty 1996; Nozawa et al. 1998). The important feature of this approach is automatic fulfillment of the first law of thermodynamics (the Gibbs-Duhem relation) (see Nozawa et al. 1998).

\section{Back-bending phenomenon, $I$ and $J$ vs. $\Omega$, and stability of rotating configurations}

The term back-bending comes from nuclear physics (see, e.g., Ring \& Shuck 1980). Nuclei can be excited by a projectile to a state of a rapid rotation corresponding to a large angular momentum quantum number $\mathcal{J}$ and excitation energy $E(\mathcal{J})$. The nuclear angular momentum is measured in the units of $\hbar$. The eigenvalues of the operator of the square of the angular momentum are $\mathcal{J}(\mathcal{J}+1)$. For $\mathcal{J} \gg 1$ one can approximate this eigenvalue by a "classical value" $\mathcal{J}^{2}$. Within the quasiclassical approximation (in which $\mathcal{J}$ can be treated as a continuous quantity) one can phenomenologically define an "angular frequency" by $\omega=\mathrm{d} E / \mathrm{d} \mathcal{J}$. The nuclear moment of inertia $\mathcal{I}$ is then found by fitting the rotational $E(\mathcal{J})$ spectra. In the quasi-classical approach, $\mathcal{I}$ is a function of $\omega$, which can be represented by a curve in the $\mathcal{I}-\omega$ plane: along this curve, $\mathcal{J}$ increases. In the standard case there is a one to one correspondence between $\mathcal{I}$ and $\omega$, and both $\mathcal{I}(\omega)$ and $\omega(\mathcal{I})$ are increasing functions of their arguments. However, for some nuclei (e.g., ${ }^{158}$ Er see Ring \& Schuck 1980) $\omega$ reaches a maximum at some value of $\mathcal{J}=\mathcal{J}_{1}$ and then decreases (back bends) to reach minimum at some larger value of $\mathcal{J}=\mathcal{J}_{2}$. At $\mathcal{J}=\mathcal{J}_{1}$ and $\mathcal{J}=\mathcal{J}_{2}$ the derivatives $\mathrm{d} \omega / \mathrm{d} \mathcal{J}$ and $\mathrm{d} \omega / \mathrm{d} \mathcal{I}$ vanish, and for $\mathcal{J}_{1}<\mathcal{J}<\mathcal{J}_{2}$ the curve $\omega(\mathcal{I})$ is "back-bending" which corresponds to $\omega$ which is decreasing with increasing $\mathcal{I}$ (see Fig. 3.4 in Ring \& Schuck 1980).

Neutron star can be treated as a huge atomic nucleus. However, such a star is a macroscopic classical object containing some $\sim 10^{57}$ baryons, to be compared with at most 200-250 nucleons in rapidly rotating nuclei. In nuclei, all high-angular-momentum states are the excited ones and therefore unstable. In the case of rotating neutron stars unstable states are not interesting, their lifetime being too short to observe them. Therefore in the case of neutron star we have to check whether a given state of stationary rotation is stable, because instability would make it astrophysically irrelevant. In this context, we find it convenient to discuss the back-bending phenomenon by studying the dependence of the total angular momentum of the star $J$ versus frequency of rotation $f=\omega / 2 \pi$. We differ in this choice from the previous work, in which the dependence $I(f)$ was studied. The reason for this choice is the following: $J$ is a well-defined quantity describing the instantaneous state of a rotating relativistic star and the evolution of the rotating star can be easily calculated under some assumptions about the change of $J$. In what follows, we restrict ourselves to evolutionary tracks of isolated neutron stars for which baryon mass $M_{\mathrm{B}}=$ const.

The moment of inertia $I$ is usually defined as $J / \Omega$ (see, e.g., Stergioulas 2003). This is the definition used in previous papers on the back-bending phenomenon in rotating neutron stars. However, such I does not describe the response of the star to the change of $J$ or $\Omega$ and therefore is not useful for checking the stability of rotating configuration. In order to obtain, e.g., the spin down of a star due to the angular momentum decrease $\mathrm{d} J$ one should have defined $\widetilde{I} \equiv \mathrm{d} J / \mathrm{d} \Omega$. Only in the slow rotation limit, where only terms linear in $\Omega$ are conserved, both definitions of the moment of inertia coincide. Total stellar angular momentum $J$ is not only a quantity with a strict physical meaning in general relativity. It also allows us to study the stability of rotating configurations with respect to axially symmetric perturbations. The point of the change in stability within a family of rotating configurations (from stable to unstable or vice versa). corresponds to the extremum of $M$ or $M_{\mathrm{B}}$ at fixed $J$ (Friedman et al. 1988):

$\left(\frac{\partial M}{\partial x}\right)_{J=\text { const }}=0, \quad\left(\frac{\partial M_{\mathrm{B}}}{\partial x}\right)_{J=\mathrm{const}}=0$, 

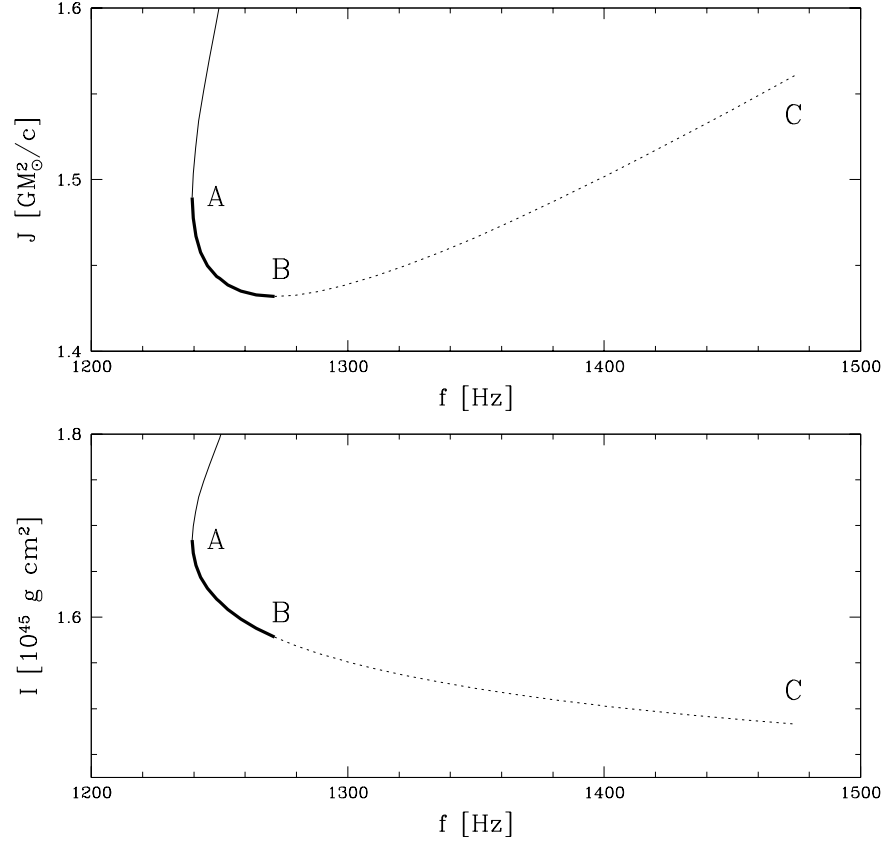

Fig. 2. The difference between the back-bending curves $J(f)$ and $I(f)$. The spin-evolution track is calculated for $M_{\mathrm{B}}=2.15 M_{\odot}$ and N2H1 EOS. Upper panel presents the dependence $J(f)$. The thicker segment $\mathrm{AB}$ represents stable back-bending evolution, while dotted segment to the right of the minimum at $\mathrm{B}$ consists of configurations unstable with respect to axisymmetric perturbation. The lower panel represents the $I(f)$ spin evolution track, considered in the previous work; the back-bending behavior occurs apparently along the whole AC branch. Actually, the BC segment of the $I(f)$ is astrophysically irrelevant, because configurations to the right of $\mathrm{B}$ are unstable.

where $x$ is the first of the two parameters which parametrize (label) stationary rotating stellar configurations (the second one being $J$ ), for example $x=\rho_{\mathrm{c}}$ or $x=P_{\mathrm{c}}$. Equivalently, the onset of instability can be determined by the condition

$\left(\frac{\partial J}{\partial x}\right)_{M_{\mathrm{B}}=\mathrm{const}}=0, \quad\left(\frac{\partial J}{\partial x}\right)_{M=\mathrm{const}}=0$.

The crucial difference between the dependencies $J(f)$ and $I(f)$ in the back-bending region is presented in Fig. 2; it will be discussed in more detail in Sect. 6. The apparently "backbending branch" in lower panel, as defined in the previous work (Glendenning et al. 1997), consists mostly of configurations unstable with respect to axial perturbations. The BC segment is astrophysically irrelevant, and the B point correspond to an unstable termination of the back-bending branch (see Sect. 7).

\section{Softening of the EOS and the baryon-mass-equatorial-radius relation at fixed rotation frequency}

Rotation modifies the relation between the baryon mass $\left(M_{\mathrm{B}}\right)$ and the circumferential equatorial radius $\left(R_{\text {eq }}\right)$ for equilibrium stellar configurations. The baryon mass $M_{\mathrm{B}}$ plays a special role, because it remains constant during the evolution of solitary pulsars. In the present section we point out specific features of the $M_{\mathrm{B}}\left(R_{\mathrm{eq}}\right)$ curves, which signal the presence of the back-bending

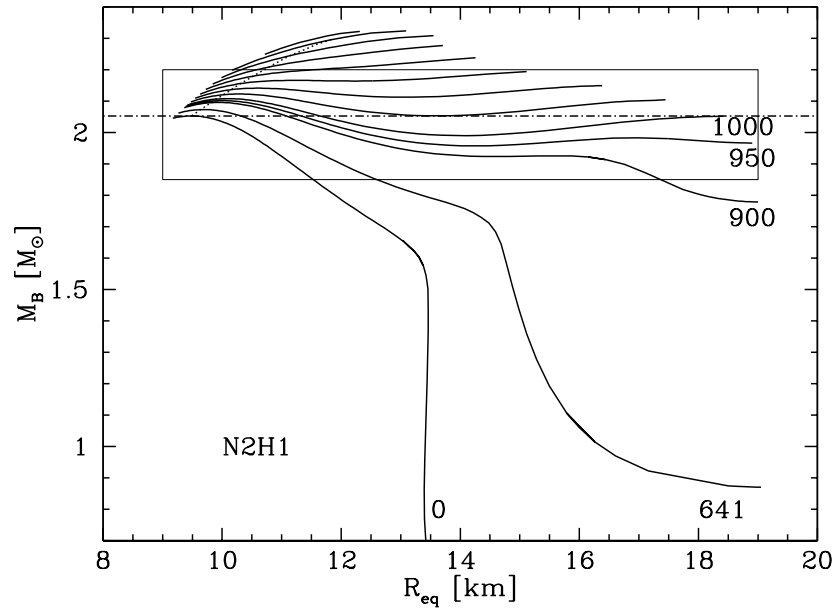

Fig. 3. Total baryon mass $M_{\mathrm{B}}$ vs circumferential equatorial radius $R_{\mathrm{eq}}$ for stationary configurations rotating at a fixed frequency, for the $\mathrm{N} 2 \mathrm{H} 1$ EOS. The curves are labeled by the rotational frequency $f=$ $\Omega / 2 \pi[\mathrm{Hz}]$. The dotted line corresponds to the onset of instability with respect to axi-symmetric (quasi-radial) perturbations. All curves terminate on the large-radius side at the mass-shedding (Keplerian) configurations. The maximum baryon mass of non-rotating stars, $M_{\mathrm{B}, \max }^{\mathrm{stat}}$, is marked by a dash-dotted line.

(BB) phenomenon in rotating neutron stars. Our calculations were performed for all the equations of state with hyperons presented in Balberg et al. (1999). However, we present detailed results (figures) only for those EOSs for which the BB phenomenon is strongly pronounced.

In Fig. 3 we show the $M_{\mathrm{B}}\left(R_{\mathrm{eq}}\right)$ curves for stars rotating at a fixed rotation frequency $f=\Omega / 2 \pi$, calculated for the $\mathrm{N} 2 \mathrm{H} 1$ EOS. An enlarged view of a particularly interesting rectangular region of the $M_{\mathrm{B}}-R_{\text {eq }}$ plane is shown in Fig. 4.

\subsection{Signature of BB: Minimum of the baryon mass at fixed frequency}

As we will show, the BB phenomenon is strictly connected to the existence of a local minimum of $M_{\mathrm{B}}$ in the $M_{\mathrm{B}}\left(R_{\mathrm{eq}}\right)$ plot at a fixed $f$. The softening of the EOS due the hyperonization leads to the flattening of the $M_{\mathrm{B}}\left(R_{\mathrm{eq}}\right)$ and $M_{\mathrm{B}}\left(\rho_{\mathrm{c}}\right)$ curves (for the case of non-rotating stars, see Balberg et al. 1999). This effect of flattening grows with increasing rotational frequency and for $f>f_{1 \text {,infx }}$ rotation may even produce a local minimum of $M_{\mathrm{B}}\left(R_{\mathrm{eq}}\right)$ and $M\left(R_{\mathrm{eq}}\right)$. The curve $M_{\mathrm{B}}\left(R_{\mathrm{eq}}\right)$ [or $M_{\mathrm{B}}\left(\rho_{\mathrm{c}}\right)$ ] at $f=f_{1, \text { infx }}$ has a very specific property. Namely, for this curve first and second derivatives of $M_{\mathrm{B}}$ with respect to the central density $\rho_{\mathrm{c}}$ vanish at some $\rho_{\mathrm{c}}=\rho_{\mathrm{c} \text {,infx }}$, i.e., the curve has there a point of inflexion which corresponds to baryon mass $M_{\mathrm{B}}^{(1, \text { infx) }}$. Our numerical calculations for the N2H1 and N1H1 EOS give $f_{1, \text { infx }} \simeq 880 \mathrm{~Hz}$ and $f_{1, \text { infx }} \simeq 860 \mathrm{~Hz}$ respectively (see Figs. 4 and 6). For $f>f_{1, \text { infx }}$ the curve $M_{\mathrm{B}}\left(R_{\mathrm{eq}}\right)$, $M_{\mathrm{B}}\left(\rho_{\mathrm{c}}\right), \ldots$, exhibit a local minimum. We find that the presence of this local minimum is an indication that for $M_{\mathrm{B}}>M_{\mathrm{B}}^{(1, \mathrm{infx})}$ the rotational evolution of neutron star with $\dot{J}<0$ exhibits a BB phenomenon in the vicinity of $f \simeq f_{1, \text { infx }}$. This is clearly seen in Figs. 4 and 6, where we show an enlargement of the vicinity 


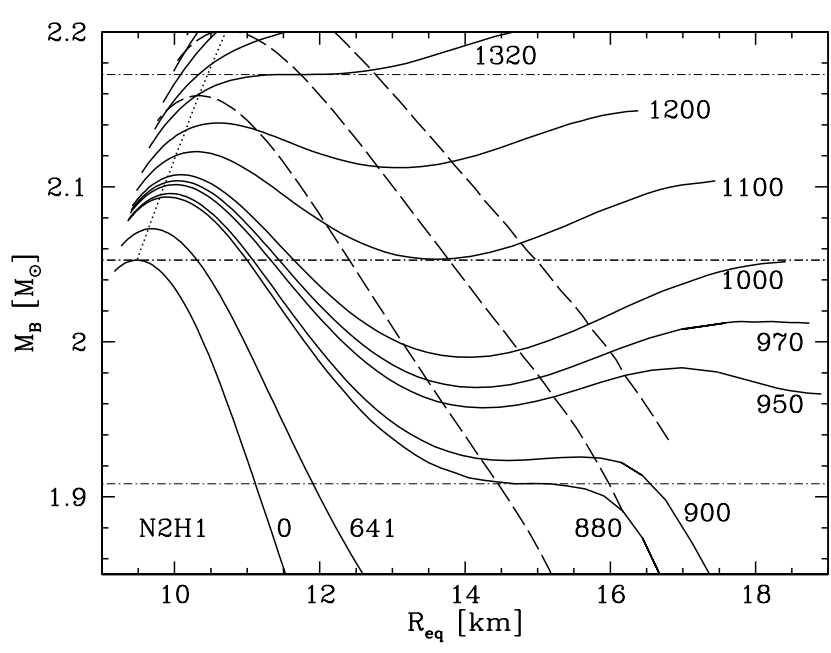

Fig. 4. The enlarged region of the back-bending phenomenon (corresponding to the box in Fig. 3). For fixed rotational frequency $f_{1, \text { infx }} \simeq$ $880 \mathrm{~Hz}$ and $f_{2, \text { infx }} \simeq 1320 \mathrm{~Hz}$ the $M_{\mathrm{B}}\left(R_{\mathrm{eq}}\right)$ dependence has an inflexion point (corresponding to the masses $M_{\mathrm{B}}^{(1, \text { infx })}=1.91 M_{\odot}$ and $M_{\mathrm{B}}^{(2, \text { infx })}=2.17 M_{\odot}$, respectively) resulting in a region where the curve is nearly flat. For $f \in\left[f_{1, \text { infx }}, f_{2, \text { infx }}\right]$ there exists a local minimum of $M_{\mathrm{B}}$. Dashed curves correspond to a fixed total angular momentum $J$. The configurations close to the local maxima are obviously stable (for a fixed $J, M_{\mathrm{B}}$ is monotonic). The evolution of an isolated star which is losing its angular momentum is represented by the motion along a horizontal line from right to the left (decreasing $J$ ). The loss of $J$ in the back-bending regime is associated with a spin up of the star.

of the inflection point, together with $J=$ const. lines. As we see, in this case there exists a range of $M_{\mathrm{B}}$ where the decrease of $J$ leads to the increase of the angular frequency which is exactly equivalent to back-bending. The fragment of the curve for which $M$ decreases as a function of $\rho_{\mathrm{c}}$ does not necessarily correspond to the instability region - the decrease of $M_{\mathrm{B}}$ at a fixed $f$ does not imply the decrease at a fixed $J$. It is only the latter condition which indicates the instability with respect to small axi-symmetric perturbations ${ }^{1}$.

In Figs. 4 and 6 we draw also three horizontal lines corresponding to fixed values of the total baryon number. A rotating star losing its angular momentum moves along horizontal line from the right to the left. The bottom lines correspond to the mass $M_{\mathrm{B}}=M_{\mathrm{B}}^{(1, \text { infx })} \simeq 1.91 M_{\odot}\left(\simeq 1.81 M_{\odot}\right)$ for the $\mathrm{N} 2 \mathrm{H} 1$ (N1H1) EOS, at which the curves for $f=f_{1, \text { infx }} \simeq$ $880 \mathrm{~Hz}(860 \mathrm{~Hz})$ have a point of inflexion. The top horizontal line corresponds to the different situation in which the curve $M_{\mathrm{B}}\left(R_{\mathrm{eq}}\right)$ has an inflexion point at a higher frequency, namely at $f=1320 \mathrm{~Hz}$ and $1100 \mathrm{~Hz}$, for the $\mathrm{N} 2 \mathrm{H} 1$ and $\mathrm{N} 1 \mathrm{H} 1$ EOSs, respectively. For baryon masses larger than the mass at this inflexion point, the angular momentum loss does not lead to the decrease of angular frequency before the onset of instability is reached, the star is all the time accelerating. The value of this limiting masses are $M_{\mathrm{B}} \simeq 2.17 M_{\odot}$ and $1.90 M_{\odot}$, respectively. The intermediate horizontal line corresponds to the maximum

${ }^{1}$ It may be shown that the configurations which realize the extrema of the $M_{\mathrm{B}}$ and $M$ at fixed $J$ coincide, see Harrison et al. (1965) for the static case, and Friedman et al. (1988) for uniformly rotating configurations.

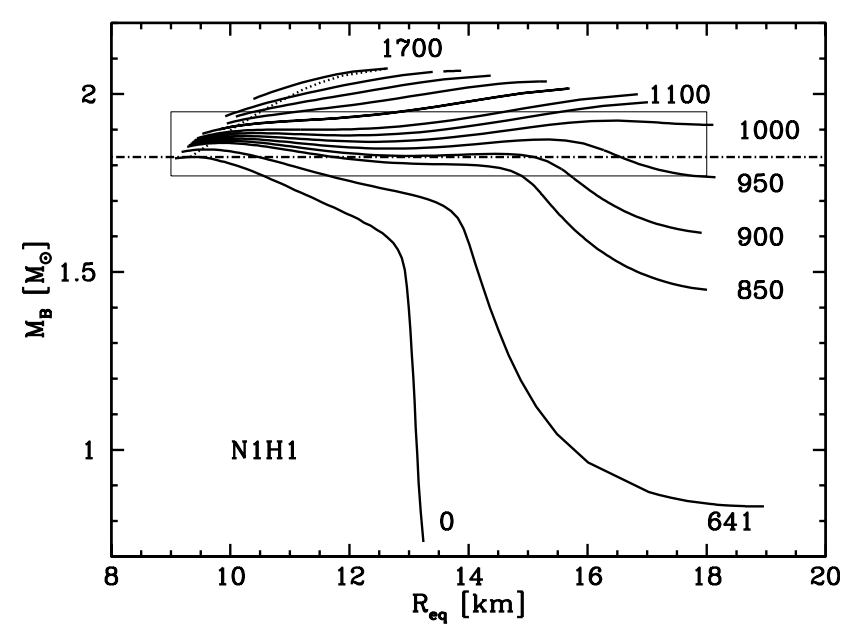

Fig. 5. Same as in Fig. 3 but for the N1H1 EOS.

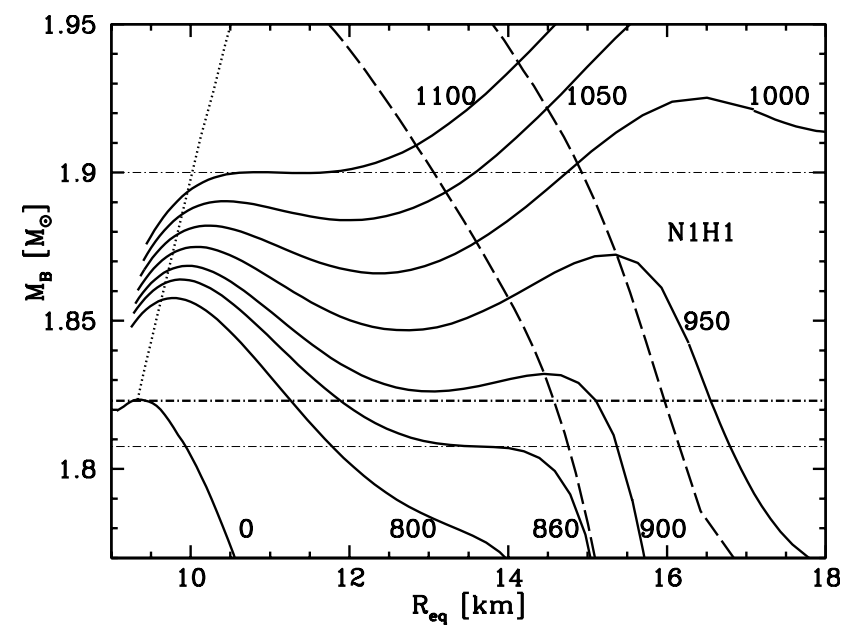

Fig. 6. The enlarged region of the back-bending phenomenon (corresponding to the box in Fig. 5).

mass of the non-rotating stars $M_{\mathrm{B}}=M_{\mathrm{B}, \text { max }}^{\text {stat }}=2.05 M_{\odot}$ and $1.824 M_{\odot}$.

\subsection{Acceleration or slowing down close to the Keplerian limit}

The interesting difference between the cases N2H1 and N1H1 concerns the behavior of the rotating star as it starts losing $J$ at the Keplerian frequency $f_{\mathrm{K}}$. The question is whether the star is then slowing down or spinning up. The actual behavior can be easily deduced from the shapes of the $M_{\mathrm{B}}\left(R_{\mathrm{eq}}\right)$ [or $\left.M_{\mathrm{B}}\left(\rho_{\mathrm{c}}\right)\right]$ curves for a fixed frequency close to the Keplerian limit. If $M_{\mathrm{B}}$ is increasing as we move in the $M_{\mathrm{B}}-R_{\text {eq }}$ plane away from the Keplerian configuration $\left[\left(\mathrm{d} M_{\mathrm{B}} / \mathrm{d} \rho_{\mathrm{c}}\right)_{\mathrm{K}}>0\right]$, the star is slowing down as it loses the angular momentum. This effect results in the S-shape of the $J(f)$ dependence in the backbending case. Otherwise $\left[\left(\mathrm{d} M_{\mathrm{B}} / \mathrm{d} \rho_{\mathrm{c}}\right)_{\mathrm{K}}<0\right]$ the isolated star is spinning up when evolving from the Keplerian configuration with $\dot{J}<0$. The limiting case corresponds to the condition $\left(\mathrm{d} M_{\mathrm{B}} / \mathrm{d} \rho_{\mathrm{c}}\right)_{\mathrm{K}}=0$ [or $\left.\left(\mathrm{d} M_{\mathrm{B}} / \mathrm{d} R_{\text {eq }}\right)_{\mathrm{K}}=0\right]$ and is represented in Fig. 4 by the curve with $f=970 \mathrm{~Hz}$ (the baryon mass at this point $\left.M_{\mathrm{B}}^{(\mathrm{K} \text {,flat })}=2.01 M_{\odot}\right)$. For the N1H1 EOS 


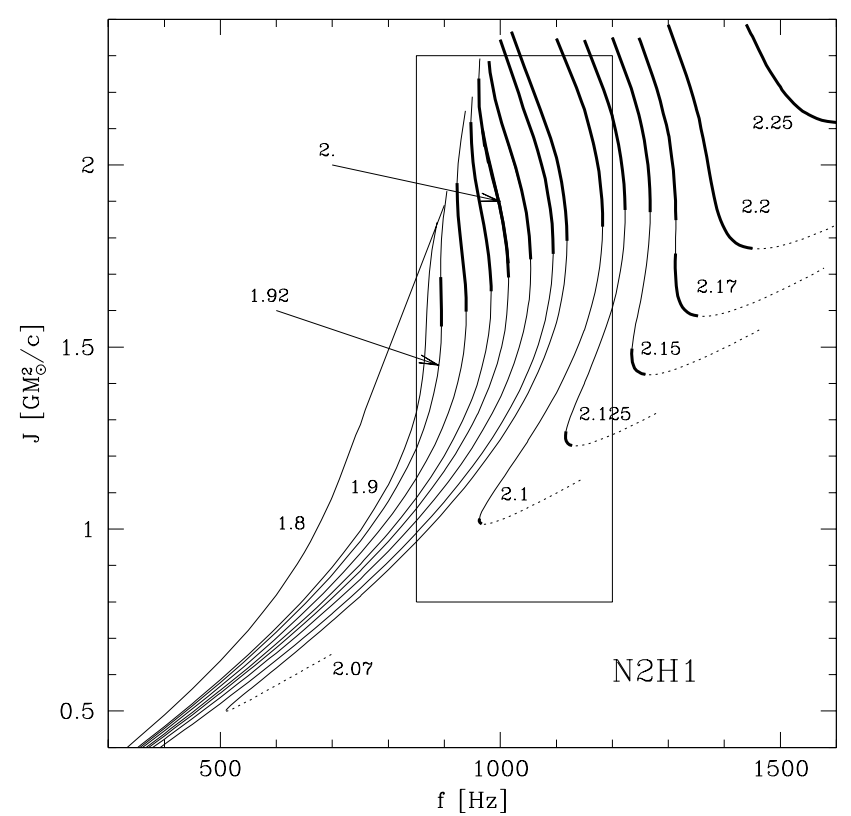

Fig. 7. Angular momentum of the star $J$ versus rotation frequency $f$, for the N2H1 EOS. Each curve corresponds to a fixed $M_{\mathrm{B}}$, whose value in $M_{\odot}$ is displayed. Along each curve, the central density increases downwards. The dotted segments correspond to configurations which are unstable with respect to axi-symmetric perturbations whereas the thick lines correspond to spin-up by angular momentum loss. An enlarged view of the rectangular region within which the back-bending occurs is shown in Fig. 8.

such an effect appears for supramassive stars $(f \simeq 1030 \mathrm{~Hz}$, $M_{\mathrm{B}}^{(\mathrm{K} \text {,flat })}=1.96 M_{\odot}$, see Fig. 6)

\section{Back-bending and stability: Analysis in the $J-f$ plane}

The search for the BB phenomenon with simultaneous testing of the stability of rotating configurations can be most conveniently carried out by plotting, at a fixed $M_{\mathrm{B}}$, the stellar angular momentum $J$ versus rotation frequency $f$. Let us start with the N2H1 EOS where the BB is the most pronounced. Several curves $J=J(f)$ at selected values of $M_{\mathrm{B}}$, calculated for this EOS, are shown in Fig. 7. These curves represent the evolution of an isolated pulsar of a given baryon mass $M_{\mathrm{B}}$, as it loses its angular momentum due to radiation of electromagnetic waves. Along each curve, the central density $\rho_{\mathrm{c}}$ increases monotonically when one moves downward. For stable configurations $J$ is a monotonic function along this path. Any minimum indicates the onset of the instability with respect to axi-symmetric perturbations. Putting it differently, for stable configurations each value of $J$ corresponds to one and only one value of $f$. The BB manifests itself as a stable segment of the $J(f)$ curve with $\mathrm{d} J / \mathrm{d} f<0$.

There is an important difference between the information one can get from the analysis of the $J(f)$ and the usually used $I(f)$ curves. Although the $I(f)$ can have segments with $\mathrm{d} I / \mathrm{d} f<$ 0 corresponding to a back-bending in a nuclear physics sense (Glendenning et al. 1997), the $I(f)$ dependence cannot tell us whether a seemingly "back-bending branch" contains

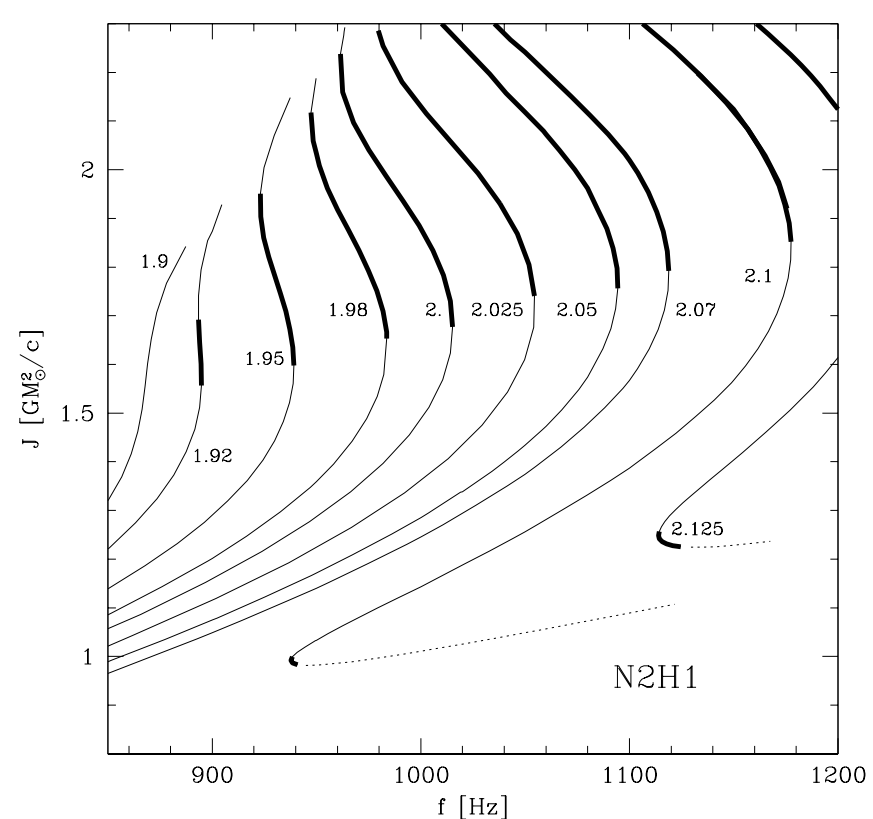

Fig. 8. Enlargement of Fig. 7.

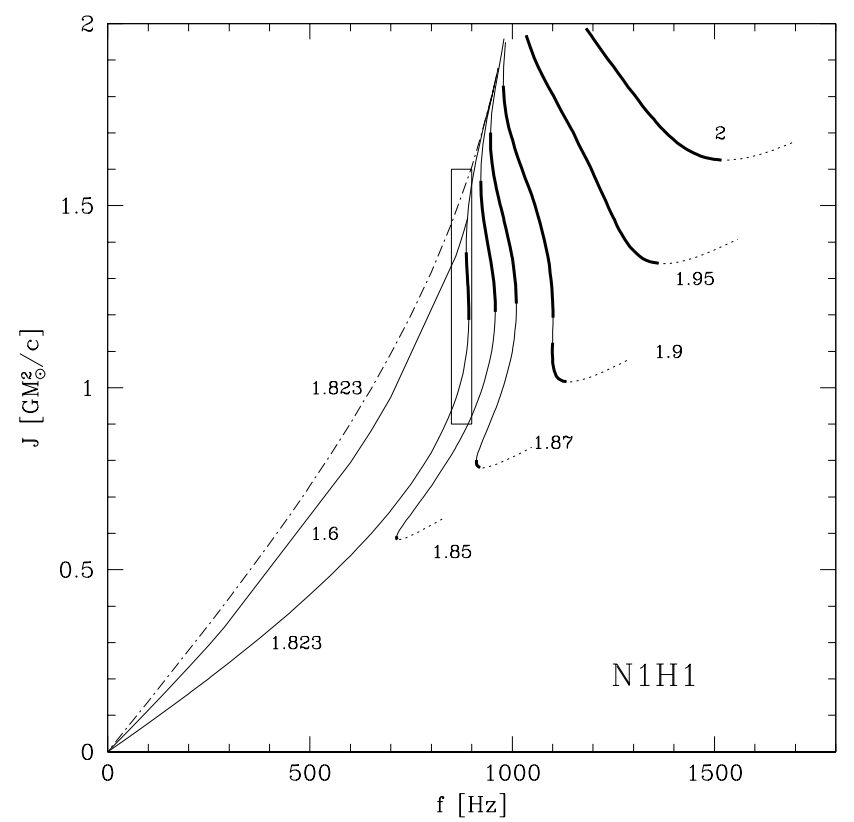

Fig. 9. Same as Fig. 7 but for N1H1 EOS. The dash-dotted line is the $J(f)$ curve for the N1 EOS (i.e., not allowing for the presence of the hyperons).

configurations which are stable. One can have a minimum in $J(f)$ on a "back-bending" segment of $I(f)$ (see Fig. 2); such a possibility was already mentioned by Spyrou \& Stergioulas (2002).

The final fate of the rotating star as its angular momentum decreases depends on $M_{\mathrm{B}}$. If $M_{\mathrm{B}}>M_{\mathrm{B}, \max }^{\text {stat }}$, the star is supramassive and eventually collapses into a black hole.

For the N1H1 EOS the BB phenomenon is less pronounced than for the $\mathrm{N} 2 \mathrm{H} 1$ one. The spin-evolution tracks at $M_{\mathrm{B}}=$ const are shown in Figs. $9[J(f)$ curves] and 10 [zoomed $J(f)$ curves in the BB region for normal configurations]. The zoomed fragment in Fig. 10 shows how narrow, compared with 


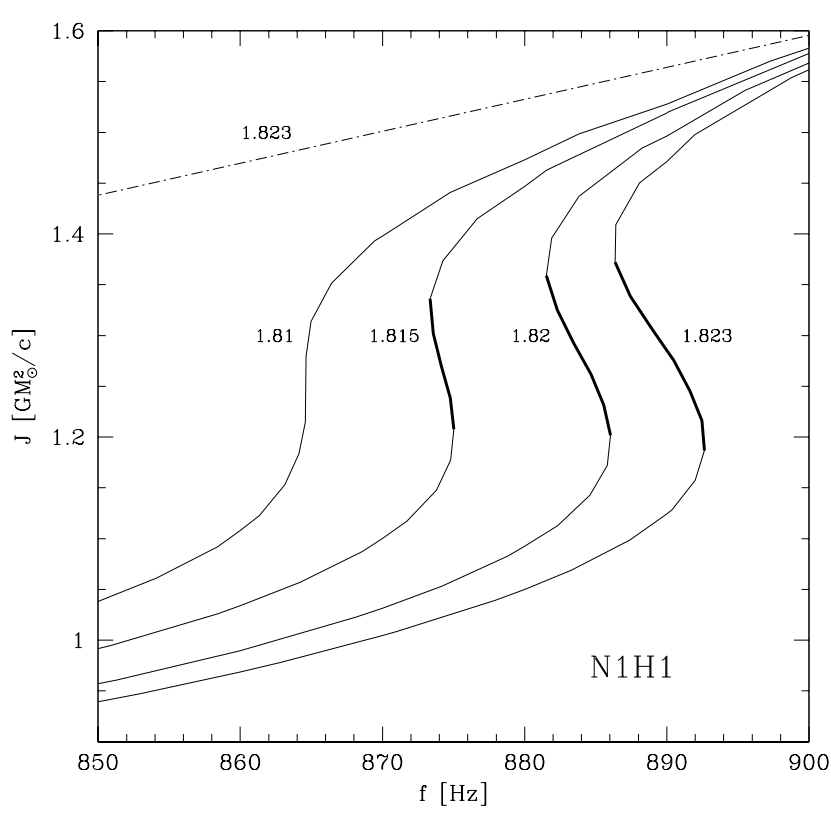

Fig. 10. Angular momentum of the star as a function of frequency for the N1H1 EOS, in the region where the back-bending phenomenon with stable termination $\left(\mathrm{BB}_{\mathrm{st}}\right.$, see the text) occurs. Each curve corresponds to a fixed value of $M_{\mathrm{B}}$ (in $M_{\odot}$ ). Along each curve, the central density increases downwards. Segments along which the angular momentum loss is associated with a spin up are indicated by a thick solid line. All configuration on the S-shaped thick segments are stable. The dash-dotted line describes a $J(f)$ trajectory for the EOS of matter in which hyperons are not allowed (N1 EOS, see the text). This curve illustrates the dramatic effect of the hyperon softening of the EOS on the pulsar spin-evolution.

the $\mathrm{N} 2 \mathrm{H} 1$ case, is the range of baryon masses for normal configurations where BB with stable termination occurs.

Using the method developed in Sect. 5, one can readily formulate the following criterion. If $M_{\mathrm{B}}^{(1, \text { infx })}>M_{\mathrm{B}, \max }^{\text {stat }}$ then the back-bending is possible only for supramassive configurations. In the opposite case, the back-bending can occur also for rotating stars with $M_{\mathrm{B}}<M_{\mathrm{B}, \max }^{\text {stat }}$.

\section{Two types of back-bending}

A look at Fig. 7 shows that there are actually two types of BB for rotating neutron stars. For isolated neutron star $\dot{J}<0$ and therefore on a stable $J(f)$ branch $J_{2}<J_{1}$ implies $t_{2}=t\left(J_{2}\right)>$ $t_{1}=t\left(J_{1}\right)$. Assume then that a BB epoch starts at $t_{1}$ and terminates at $t_{2}$. For the first type of BB, occurring for the baryon mass $2.25 M_{\odot}$ and $2.2 M_{\odot}$ for the N2H1 EOS (Fig. 7), and for $2 M_{\odot}$ and $1.95 M_{\odot}$ for the N1H1 EOS (Fig. 9), the point at $t_{2}$ corresponds to an unstable rotating configuration. Such a BB with an unstable termination will be denoted as $\mathrm{BB}_{\mathrm{ut}}$. This type of $\mathrm{BB}$ occurs above some value of $M_{\mathrm{B}}$ equal to $M_{\mathrm{B}}^{(2, \mathrm{infx})}$. An isolated neutron star enters the $\mathrm{BB}$ regime but it will not reappear in the normal spin-down regime with $\mathrm{d} J / \mathrm{d} f>0$. However, the situation changes as decreasing $M_{\mathrm{B}}$ crosses the value $M_{\mathrm{B}}^{(2, \text { infx) }}$. Then the BB segment splits into two segments. The segment with higher $J$ (corresponding to an earlier epoch) terminates at a stable configuration, and will be denoted by $\mathrm{BB}_{\mathrm{st}}$. Then follows an epoch of normal spin down with $\mathrm{d} J / \mathrm{d} f>0$, followed by a $\mathrm{BB}_{\mathrm{ut}}$ terminated by an instability. With decreasing $M_{\mathrm{B}}$, the $\mathrm{BB}_{\mathrm{ut}}$ epoch becomes shorter and shorter, and becomes infinitesimally small as one reaches the maximum mass of nonrotating configuration $M_{\mathrm{B} \text {,max }}^{\text {stat }}$. For baryon masses larger than $M_{\mathrm{B}}^{(1, \text { infx })}$ we are dealing with a "BB episode" in an otherwise normal neutron star rotational evolution.

The case of $\mathrm{BB}_{\mathrm{ut}}$ can be quite easily discussed on the basis of the $M_{\mathrm{B}}\left(R_{\mathrm{eq}}\right)$ dependence (for example Fig. 4). The $\mathrm{BB}_{\mathrm{ut}}$ phenomenon corresponds to a specific location of the instability line (dotted line in Fig. 4) with respect to the maxima of the functions $M_{\mathrm{B}}\left(R_{\mathrm{eq}}\right)$ at fixed $f$ (if they exist). Because these maxima are to the right of the instability line (i.e., in the stable region) $\mathrm{BB}_{\mathrm{ut}}$ always appears. In the other words, a star with fixed $M_{\mathrm{B}}$, approaching the instability point, has to eventually spin up. This conclusion follows immediately from a Lemma formulated by Friedman et al. (1988) ${ }^{2}$. These authors considered a two-parameter family of uniformly rotating stars with a oneparameter EOS $P=P(\rho)$. In general, a continuous sequence of rotating configurations can be labeled by a parameter $\lambda$ so that along this sequence all stellar parameters are functions of $\lambda$. In order to avoid confusion with time derivative of a stellar quantity $Q$, we will denote a derivative of $Q$ with respect to $\lambda$ by $Q^{\prime} \equiv \mathrm{d} Q / \mathrm{d} \lambda$. According to Friedman et al. Lemma, the unstable region corresponds to the part of the sequence $J(f)$ for which $J^{\prime} f^{\prime}>0$ (since along these sequences $M_{\mathrm{B}}^{\prime}=0$ ), or equivalently, $\mathrm{d} J / \mathrm{d} f>0$, which means that just before the instability is reached one has $\mathrm{d} J / \mathrm{d} f<0$, i.e., the spin up by the angular momentum loss.

Concluding, $\mathrm{BB}_{\mathrm{ut}}$ is a feature of any equation of state. However the significance of this effect depends on the stiffness of the matter. In contrast $\mathrm{BB}_{\mathrm{st}}$ exists only when $M_{\mathrm{B}}$ has a local minimum for fixed rotational frequency.

The impact of the hyperon EOS softening on the character and significance of the BB phenomenon can be clearly appreciated by comparing Figs. 7 and 9 with Fig. 11 obtained for the (nucleonic) N1 EOS without hyperons. For the nucleonic EOS, BB is present only for supramassive configuration and it is significant only for relatively high masses (although strictly speaking it is present for all masses larger than $M_{\mathrm{B}, \max }^{\text {stat }}-$ for masses $M_{\mathrm{B}}<2.6 M_{\odot}$ the $\mathrm{BB}_{\mathrm{ut}}$ region is so narrow that it is not visible in Fig. 11), see Cook et al. (1994) and Salgado et al. (1994). In contrast, in the case of the EOS with hyperons, the $\mathrm{BB}$ is present for configurations close to the Keplerian one for very broad range of masses. The $\mathrm{BB}$ epoch for the nucleonic EOS terminates always by an instability.

\section{The EOS-dependence of the back-bending phenomenon}

The specific form of the BB phenomenon depends on the degree of softening of the EOS above the hyperon threshold. We considered a rather large set of the hyperonic matter, including four models of Balberg \& Gal (1997) described in Sect. 2, and the five models of Glendenning (1985). Rapid rotation and

\footnotetext{
2 We note a misprint in this Lemma text as printed in Friedman et al. (1988), who use dots to denote a derivative with respect to $\lambda$ : the dot over the right bracket in $(\dot{\Omega} \dot{J}+\dot{\mu} \dot{N}) \neq 0$ is missing.
} 


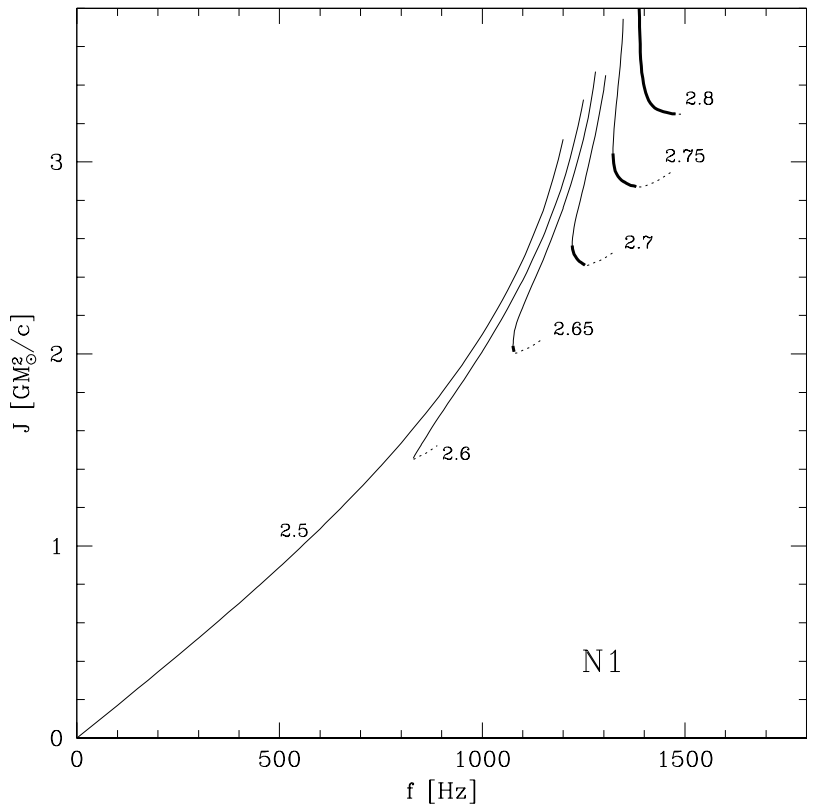

Fig. 11. Angular momentum of a star with fixed baryon mass $M_{\mathrm{B}}$ as a function of frequency for the N1 EOS. Segments constraining unstable configurations are dotted, those corresponding to the spin-up by the angular momentum loss are indicated by a thick solid line.

spin-evolution for the N1H1 and N2H1 EOSs of Balberg \& Gal (1997) and of an EOS of Glendenning (1985), were previously studied, using different numerical method of solving of the 2-D equations of hydrostatic equilibrium, by Balberg et al. (1999).

We obtain the most pronounced BB behavior for the N2H1 EOS, which was not studied by Balberg et al. (1999). In this model the nucleonic EOS is very stiff, and the hyperon softening is not so strong as for the EOS with the H2 hyperonic sector. Such features are optimal for getting a strong BB effect for a large interval of stellar baryon mass. In particular, we get $\mathrm{BB}_{\mathrm{st}}$ for a relatively large interval of normal rotating configurations with $M_{\mathrm{B}}=(1.91-2.05) M_{\odot}$, which extends up to $2.17 M_{\odot}$ if supramassive rotating models are also included. At $M_{\mathrm{B}} \simeq 2 M_{\odot}$ the star which starts its spin evolution at $f \simeq f_{\mathrm{K}}$ accelerates its rotation by $\sim 50 \mathrm{~Hz}$ during the first epoch of its evolution when nearly half of its initial angular momentum is lost ! Implications of such a substantial "back-bending with stable termination" for the pulsar timing interpretation will be discussed in Sect. 9.

The N1H1 EOS has significantly softer nucleon component, and more important flattening of the $M_{\mathrm{B}}\left(R_{\mathrm{eq}}\right)$ curve. As for the N2H1 EOS, the $\mathrm{BB}_{\mathrm{st}}$ phenomenon occurs not only for the supramassive configurations but also for the normal ones, with $M_{\mathrm{B}}<M_{\mathrm{B}, \max }^{\text {stat }}$ (Fig. 9). However, the range of the baryon masses of the normal configuration for which the $\mathrm{BB}_{\text {st }}$ occurs is very narrow, $M_{\mathrm{B}}^{(1, \text { infx })}=1.81 M_{\odot}<M_{\mathrm{B}}<M_{\mathrm{B}, \max }^{\text {stat }}=1.823 M_{\odot}$. For such baryon masses $\mathrm{BB}_{\text {st }}$ takes place at $f>f_{1 \text {,infx }} \simeq$ $850 \mathrm{~Hz}$. The $\mathrm{BB}_{\mathrm{st}}$ itself for this EOS is due to the high degree of flatness of the hyperon section of the $M_{\mathrm{B}}\left(R_{\mathrm{eq}}\right)$ plot. However, due to this flatness the values of $M_{\mathrm{B}}^{(1, \text { infx })}$ and $M_{\mathrm{B}, \max }^{\mathrm{stat}}$ are also very close to each other. All in all, even including the

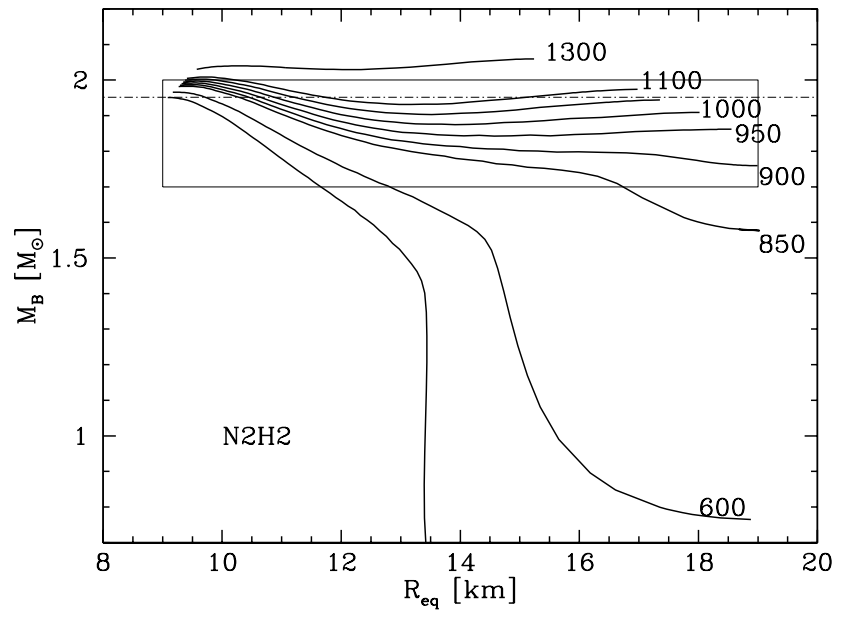

Fig. 12. Model N2H2. Baryon-mass versus circumferential equatorial radius for fixed frequency. The minimum for fixed $M_{\mathrm{B}}$ appears approximately at the same frequency at which the decreasing part for large $R_{\text {eq }}$ (close to Keplerian configurations) disappears, i.e. this limiting case correspond to the nearly flat curve $\left(M_{\mathrm{B}}=\right.$ const) up to the Keplerian configuration. There is no maximum for fixed frequency.

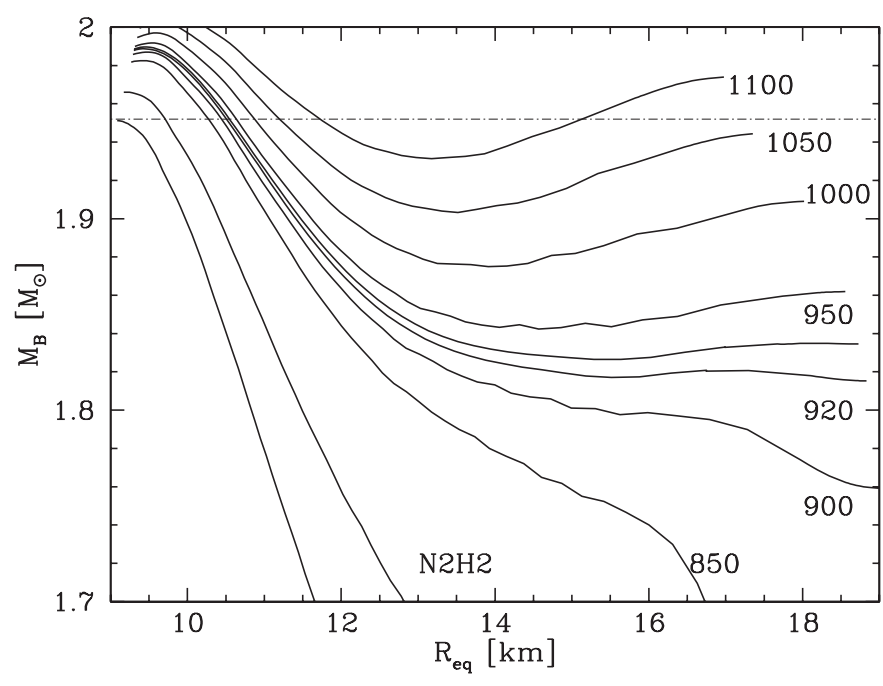

Fig. 13. Model $\mathrm{N} 2 \mathrm{H} 2$. The enlarged region marked by the rectangular box in Fig. 12. For $f \simeq 920 \mathrm{~Hz}$ there exist region where the curve is nearly flat $\left(M_{\mathrm{B}}\right.$ does not change) up to the Keplerian configurations. For higher $f$ there exist a minimum of $M_{\mathrm{B}}$. The evolution of the isolated star which is losing its angular momentum is represented by the motion along horizontal line from right to the left (decreasing $J$ ). For $M_{\mathrm{B}}>1.82 M_{\odot}$ angular momentum loss leads to spin up. For explanation of the "way features" of most curves see the text.

supramassive configurations, the range of baryon masses for which the $\mathrm{BB}_{\text {st }}$ occurs is not large, $1.81 M_{\odot}<M_{\mathrm{B}}<1.9 M_{\odot}$.

The situation changes if we consider the N2H2 EOS (see Sect. 2), where the hyperon softening is stronger. Let us repeat the analysis applied previously to N1H1 EOS in Sect. 5. The $M_{\mathrm{B}}\left(R_{\text {eq }}\right)$ curves at several values of $f$ are shown in Fig. 12. In contrast to the case of the N1H1 EOS, pictured in Fig. 5 there is no curve with a visible second (large-radius) maximum. A zoom of the relevant baryon-mass-equatorial-radius rectangle is displayed in Fig. 13. A marginal second maximum appears at $f \simeq 920 \mathrm{~Hz}$. However, this second maximum is not a robust 
Table 1. Main parameters relevant for the spin evolution of isolated neutron stars with hyperons. The labels "infx" mark the configurations for which the curve $M_{\mathrm{B}}\left(R_{\mathrm{eq}}\right)_{f}$ has a point of inflexion. For $f_{1, \text { infx }}<f<f_{2, \text { infx }}$ (corresponding masses $M_{\mathrm{B}}^{(1, \text { infx) }}$ and $M_{\mathrm{B}}^{(2, \text { infx })}$ ) $\mathrm{BB}_{\mathrm{st}}$ exists. For $M_{\mathrm{B}}>M_{\mathrm{B}, \max }^{\mathrm{stat}}$ we have also $\mathrm{BB}_{\mathrm{ut}}$, which is the only $\mathrm{BB}$ phenomenon for $M_{\mathrm{B}}>M_{\mathrm{B}}^{(2, \text { infx })}$. For $f>f_{\mathrm{K}, \text { flat }}$ and $M_{\mathrm{B}}>M_{\mathrm{B}}^{(\mathrm{K}, \text { flat })}$, a star which is initially in the mass-shedding (Keplerian) state, spins-up as it looses angular momentum. The characteristic S-shape of $J(f)$ is then impossible.

\begin{tabular}{ccccc}
\hline \hline Parameter & $\mathrm{N} 1 \mathrm{H} 1$ & $\mathrm{~N} 2 \mathrm{H} 1$ & $\mathrm{~N} 1 \mathrm{H} 2$ & $\mathrm{~N} 2 \mathrm{H} 2$ \\
\hline$M_{\mathrm{B}, \text { max }}^{\text {stat }}\left[M_{\odot}\right]$ & 1.83 & 2.05 & 1.73 & 1.95 \\
\hline$f_{1, \text { infx }}[\mathrm{Hz}]$ & 860 & 880 & 880 & 910 \\
$M_{\mathrm{B}}^{(1, \text { infx }}\left[M_{\odot}\right]$ & 1.808 & 1.91 & 1.73 & 1.81 \\
\hline$f_{\mathrm{K}, \mathrm{flat}}[\mathrm{Hz}]$ & 1030 & 970 & 1050 & 930 \\
$M_{\mathrm{B}}^{\text {(K,flat }}\left[M_{\odot}\right]$ & 1.96 & 2.01 & 1.87 & 1.82 \\
\hline$f_{2, \text { infx }}[\mathrm{Hz}]$ & 1100 & 1320 & 1080 & 1380 \\
$M_{\mathrm{B}}^{(2, \text { infx }}\left[M_{\odot}\right]$ & 1.9 & 2.17 & 1.79 & 2.06 \\
\hline
\end{tabular}

one. It can be detected only if the precision of the calculation is sufficiently high. This is to be contrasted with the N1H1 case, where the second maxima on the $M_{\mathrm{B}}-R_{\mathrm{eq}}$ curves are well pronounced, see Fig. 6. Let us notice that $\mathrm{N} 2 \mathrm{H} 2$ is the EOS for which the BB phenomenon for normal configurations was detected by Balberg et al. (1999).

The main features of the dependence of the back-bending phenomenon on the EOS can be summarized on the basis of the Table 1 containing relevant parameters for considered EOSs. Let us start with four characteristic baryon masses $M_{\mathrm{B}}^{(1, \mathrm{infx})}$, $M_{\mathrm{B}}^{(2, \text { infx })}, M_{\mathrm{B}}^{(\mathrm{K} \text {,flat })}, M_{\mathrm{B}, \max }^{\text {stat }}$. Within this set, the ordering according to the baryon-mass value turns out to depend only on the pure nucleon EOS. The sequence for N1H1 and N1H2 EOSs is: $M_{\mathrm{B}}^{(1, \text { infx })}<M_{\mathrm{B}, \max }^{\text {stat }}<M_{\mathrm{B}}^{(2, \text { infx })}<M_{\mathrm{B}}^{(\mathrm{K}, \text { flat })}$ whereas for the $\mathrm{N} 2 \mathrm{H} 1$ and N2H2 EOSs we have $M_{\mathrm{B}}^{(1, \text { infx })}<M_{\mathrm{B}}^{(\mathrm{K}, \text { flat })}<M_{\mathrm{B}, \max }^{\text {stat }}<$ $M_{\mathrm{B}}^{(2, \text { infx) }}$. As can be seen the main difference is a rather low value of $M_{\mathrm{B}}^{(\mathrm{K}, \text { flat })}$ for the $\mathrm{N} 1 \mathrm{H} 1$ and N1H2 models. In the case of the N2 model of the nucleon component, rotation changes the properties of the star close to the Keplerian frequency much more effectively than for the $\mathrm{N} 1$ one.

The nucleon N2 EOS is stiffer than the N1 one. As a result, the stars with nucleon envelopes based on the N2 model are more extended than the $\mathrm{N} 1$ ones, having larger radius at the same mass. On the other hand, the density profiles in the nucleon envelopes of the $\mathrm{N} 2 \mathrm{H}(\mathrm{H}=\mathrm{H} 1, \mathrm{H} 2)$ stars have smaller radial gradients, and therefore they play more important role during rotation. For example, the N2 envelopes have significantly larger mass than the $\mathrm{N} 1$ ones. Consequently, a smaller rotational frequency $\left(f_{\mathrm{K} \text {,flat }}=930,970 \mathrm{~Hz}\right.$ for $\mathrm{H} 2, \mathrm{H} 1$ softening, respectively), than in the case of the N1 model, is needed to make the effects of rotation so important that the maximum of $M$ close to Keplerian frequency disappears. This effect can be easily seen in Table 1 where not only the N1H frequencies $f_{\mathrm{K} \text {,flat }}$ are significantly larger than the $\mathrm{N} 2 \mathrm{H}$ ones, but also the mass $M_{\mathrm{B}}^{(\mathrm{K}, \text { flat })}$ is significantly larger. Not only it exceeds the maximum mass for the nonrotating stars, $M_{\mathrm{B} \text {,max }}^{\text {stat }}$, but it is even larger than $M_{\mathrm{B}}^{(2, \text { infx })}$. In this case the $M_{\mathrm{B}}^{(2, \text { infx })}$ determines the mass above which only spin up by angular momentum loss is possible.

As the N1 EOS is softer than the N2 one, the additional softening by hyperons leads almost immediately to the maximum mass of nonrotating configurations; the hyperon segment beyond the "hyperon knee" is very flat. The back-bending phenomenon $\mathrm{BB}_{\mathrm{st}}$ is present between the two inflexion points, and in general is not connected with the maximum mass of nonrotating stars.

The $M_{\mathrm{B}}\left(R_{\mathrm{eq}}\right)$ curves displayed in Fig. 13 deserve an additional comment, referring to the precision of the 2-D calculations. Nearly all curves for $f=850-1100 \mathrm{~Hz}$ (except for two curves for $f \simeq 920 \mathrm{~Hz}$ ) were obtained in numerical calculations in which the innermost zone boundary is not adjusted to the surface of the hyperon threshold. Consequently, these curves exhibit "wavelets" which result from an insufficient precision of the numerical calculations. In contrast, the two curves for $f \simeq 920 \mathrm{~Hz}$ are calculated with the innermost zone boundary at the hyperon-softening threshold, which enables a much higher precision.

The division of the stellar interior into two zones is particularly effective in the case of a strong change in the $\operatorname{EOS} P(\rho)$ at some density. In our case we have a rather stiff EOS below the threshold density for the hyperon appearance, and a soft EOS for dense matter with hyperons. Therefore, we put the inner zone boundary at the threshold of hyperon appearance, where the adiabatic index of the EOS suffers a significant drop. This allows us for very accurate calculation by the spectral method also in the region close to the hyperon threshold. Although in our case we do not encounter a density jump at the zone boundary, our method can be also used for an EOS with a density jump due to a first-order phase transition.

\section{Discussion and conclusion}

The presence of hyperons in neutron-star cores can strongly affect the spin evolution of a solitary neutron star (e.g., an isolated pulsar). As we have shown, such a neutron star can live a long epoch of "spin down by the angular momentum loss", and this could occur for a broad range of baryon mass of neutron star. The epoch with $\dot{P}<0$ despite $\dot{J}<0$ could terminate by an instability or by a stable continuous transition to a "standard" spin-down epoch.

We paid particular attention to the $\left(\dot{P}<0, \dot{J}<0, M_{\mathrm{B}}=\right.$ const) epoch with a stable termination. It is represented by an S-shaped segment of the spin-evolution track in the $J-f$ and $I-f$ planes, and was baptized "back-bending phenomenon" in the previous literature. Various regimes of the spin evolution were shown to be correlated with the behaviour of the $M_{\mathrm{B}}\left(R_{\mathrm{eq}}\right)_{f}$ curves at fixed $f$. In particular, we pointed out importance of the location of the inflection points of the $M_{\mathrm{B}}\left(R_{\mathrm{eq}}\right)$ curves for the existence of the back-bending phenomenon. We were also inclined to leave the name of back-bending only to the evolution-track segments with a stable termination $\left(\mathrm{BB}_{\mathrm{st}}\right.$ in our terminology).

Epochs with back-bending for normal rotating configurations were found for two of the four EOS of Balberg \& Gal (1997). On the other hand, we found that the back-bending 
phenomenon for normal sequences was absent for five hyperonic EOSs of Glendenning (1985). This illustrates the uncertainties in the hyperonic EOS, stemming from a high degree of ignorance concerning the nucleon-hyperon and particularly hyperon-hyperon interactions in dense matter.

Throughout this paper we stressed that the back-bending in the $I-f$ plane, considered up to now in the literature, should be accompanied by stability analysis; only stable back-bending configurations are astrophysically relevant. We performed such a stability analysis, and we found that very often dominant back-bending segments of the $I(f)_{\mathrm{M}_{\mathrm{B}}}$ are unstable with respect to the axisymmetric perturbations, and therefore do not exist in the Universe.

As it has been mentioned by Spyrou \& Stergioulas (2002), in such kind of calculations it is extremely important to assure the thermodynamical consistency of the EOS (the first law of thermodynamics has to be strictly fulfilled). It is well known that a rough treatment of this condition can lead to an inaccurate determination of the maximum mass and stability conditions (for example the configurations corresponding to the maximum of $M$ and $M_{\mathrm{B}}$ do not coincide - this is an evidence for a lack of the thermodynamical consistency in the EOS).

The precision of the code is also very important and in our case the proper division of the star into two computational domains (at the threshold for appearance of hyperons) allows us to obtain high precision results without a large increase of the number of grid points.

An isolated pulsar, born in a SN II explosion, could have an interesting and nonstandard past due to a hyperon softening of the EOS. As we showed, such a pulsar could lose some half of its initial angular momentum without changing much its rotation period. Therefore, if one observes a rapid pulsar with a characteristic age $\tau_{\text {PSR }}$ significantly longer than the age of a supernova remnant where this pulsar is born, $\tau_{\mathrm{SNR}}$, this might be due to some back-bending episode, resulting from a (hyperon? phase-transition?) softening of the EOS of its core. Such a possibility of explaining a seeming contradiction between $\tau_{\text {SNR }}$ and $\tau_{\text {PSR }}$ has been already noticed, in the context of a mixed-phase EOS softening, by Spyrou \& Stergioulas (2002). Clearly, the observational pulsar-timing signatures of the EOS softening due to the hyperon or phase-transition softening of the EOS deserve further studies, and we are planning to continue such studies using our high-precision 2-D code.

Another interesting consequence of the hyperon softening of the EOS could be a "period clustering" of rotating neutron stars powered by accretion in the long living low-mass X-ray binaries. This problem is now being studied.

Acknowledgements. We are very grateful to Nick Stergioulas for helpful correspondence on the spin evolution of solitary neutron stars, and for making possible comparison of numerical results obtained by our two groups. We express our gratitude to John L. Friedman, who during a car ride from Orsay to Meudon gave us precious advice concerning the axisymmetric instabilities in rotating neutron stars. Last but not least, we thank Brandon Carter whose careful driving made such a fruitful discussion possible. This work was partially supported by KBN grants 5P03D.020.20 and 2P03D.019.24.

\section{References}

Ambartsumyan, V. A , \& Saakyan G. S 1960, Astron. Zhur., 37, 193 (English translation in Sov. Astron. AJ, 4, 187)

Balberg, S., \& Gal, A. 1997, Nucl. Phys. A, 625, 435

Balberg, S., Lichtenstadt, I., \& Cook, G. P 1999, ApJ, 121, 515

Bonazzola, S., Gourgoulhon, E., \& Marck, J.-A. 1998, Phys. Rev. D, 58, 104020

Bonazzola, S., Gourgoulhon, E., Salgado, M., \& Marck, J.-A. 1993, A\&A, 278, 421

Cameron, A. G. W 1959, ApJ, 129, 676

Cheng, K. S., Yuan, Y. F., \& Zhang, J. L. 2002, ApJ, 564, 909

Chubarian, E., Grigorian, H., Poghosyan, G., \& Blaschke, D. 2000, A\&A, 357, 968

Cook, G. B., Shapiro, S. L., \& Teukolsky, S. A. 1994, ApJ, 423, L117

Friedman, J. L., Ipser, J. R, \& Sorkin, R. D. 1988, ApJ, 325, 722

Glendenning, N. K. 1985, ApJ, 293, 470

Glendenning, N. K., Pei, S., \& Weber, F. 1997, Phys. Rev. Lett., 79, 1603

Glendenning, N. K. 2001, J. Phys. G: Nucl. Part. Phys., 28, 2023

Glendenning, N. K., \& Weber, F. 2002, AIP Conf. Proc., 610, 470

Gourgoulhon, E., Haensel, P., Livine, R., et al. 1999, A\&A, 349, 851

Haensel, P. 2003, in Final Stages of Stellar Evolution, ed. J.-M. Hameury, \& C. Motch (EDP Sciences), EAS Publ. Ser., 7, 249

Haensel, P., Levenfish, K. P., \& Yakovlev, D.G. 2002, A\&A, 394, 213

Nozawa, T., Stergioulas, N., Gourgoulhon, E., \& Eriguchi, Y. 1998, A\&AS, 132, 431

Ring, P., \& Schuck, P. 1980, The Nuclear Many Body Problem (Berlin: Springer)

Salgado, M., Bonazzola, S., Gourgoulhon, E., \& Haensel, P. 1994, A\&A, 291, 155

Salpeter, E. E. 1960, Ann. Phys., 11, 393

Spyrou, N. K., \& Stergioulas, N. 2002, A\&A, 395, 151

Stergioulas, N. 2003, Living Rev. Relativity, 6, 3, e-mail: http://www . livingreviews.org/lrr-2003-3

Swesty, F. D. 1996, J. Comp. Phys., 127, 118 